\title{
Eléments pour une sociologie des études féministes en Europe centrale et orientale*
}

Ioana Cîrstocea

Le domaine de recherche et d'enseignement des women's/gender/feminist studies ${ }^{1}$ s'est institutionnalisé dans les pays de l'Europe centrale et orientale (PECO) pendant la décennie 1990. Les travaux rangés sous ces labels fonctionnent souvent à des fins d'expertise, se donnent pour but de contribuer à améliorer la condition des femmes et se situent au cœur des programmes de recomposition sociale et politique lors de la sortie du communisme. Mises en place par des agents qui investissent simultanément des espaces sociaux contrastés (associatif, scientifique et politique), ces études se trouvent au croisement de logiques savantes et militantes, nationales et internationales. Cela en fait un révélateur de la refonte des sciences sociales, de l'émergence de nouveaux champs d'interrogations, de la circulation transnationale des problématiques scientifiques, enfin, des recompositions des élites savantes dans l'espace ex-soviétique. La sociologie de la constitution de ce nouveau domaine de connaissances est à faire ${ }^{2}$ : étude systématique des agents qui se trouvent à son origine, analyse de la genèse des discours savants qu'ils véhiculent ainsi que du fonctionnement du féminisme «de la globalisation», dont les logiques pratiques sont encore peu étudiées bien

\footnotetext{
* Notre gratitude va à Francine Muel-Dreyfus, Martine Mespoulet, Michèle Ferrand, Delphine Naudier, Françoise Battagliola, Bernard Pudal, Rose-Marie Lagrave et Christiane Veauvy, pour leur lecture critique ainsi que pour les encouragements portés à ce travail depuis plusieurs années. Nous remercions également le Gender Studies Department de la Central European University de Budapest pour son accueil à l'occasion d'un séjour de recherche effectué au second semestre 2007-2008.

${ }^{1}$ L'intitulé varie d'un pays à l'autre et sous-entend une diversité de positions politiques allant de l'engagement féministe affiché à la volonté de masquer ce même engagement par l'adoption du terme anglais " gender », supposé avoir une résonance à la fois « scientifique », « neutre » et « moderne » (cf. R. Braidotti, 1997; 2002 sur les «politiques de la dénomination»).

${ }^{2}$ Cf. S. Zimmermann, 2007, pour une des rares études synthétiques consacrées à l'institutionnalisation des gender studies dans l'espace ex-soviétique.
} 
qu'il soit reconnu comme une réalité des dernières décennies par la plupart des travaux d'histoire des mouvements de femmes ${ }^{3}$.

\section{« Effets sociaux» des transformations postcommunistes, femmes « victimes » et}

\section{offre féministe}

Les bilans des recherches consacrées aux PECO, devenus nombreux depuis le tournant des années 2000, mettent en lumière la fortune, heureuse ou malheureuse, des divers concepts et paradigmes explicatifs successivement mobilisés, tout en faisant état du défi épistémologique posé par les dimensions sociales des transformations postcommunistes, devant lesquelles les spécialistes en sciences sociales se sont avérés assez démunis. Quelques propositions heuristiques entendent pallier les insuffisances des approches transitologiques et néofonctionnalistes, prédominantes pendant la décennie 1990 : étude des reconversions des acteurs individuels et collectifs, analyse de la continuité des structures sociales avant et après le changement de régime politique, connaissance fine de l'histoire sociale des régimes communistes comme préalable épistémologique à toute recherche sur le postcommunisme ${ }^{4}$. Toutefois, rares sont les travaux qui échappent à la logique selon laquelle les «effets »ou « coûts sociaux » des réformes sont réduits à leur «contexte», donnés pour secondaires et renvoyés en fin d'ouvrage, ou bien abordés de façon schématique sous l'angle de l'apparition de catégories défavorisées et du partage du monde social entre « gagnants » et «perdants ».

\footnotetext{
${ }^{3}$ Promu par les organismes supranationaux (notamment l'Organisation des Nations unies, mais aussi l'Union européenne), le féminisme «interétatique» est institué par des moyens bureaucratiques et consiste principalement à faire reconnaître et défendre les «droits humains des femmes » (H. Hirata et alii, 2000 ; N. A. Naples, M. Desai, 2002 ; E. Gubin et alii, 2004 ; P. Antrobus, 2004 ; M. Desai, 2005 ; M. M. Ferree, A. M. Tripp, 2006).

${ }^{4}$ Revue française de science politique (« Les transitions démocratiques. Regards sur la 'transitologie' »), 50, 4-5, 2000 ; C. M. Hann, 2002; D. Colas, 2002 ; M. Forest, G. Mink, 2004 ; S. Kott et alii, 2006.
} 
La littérature cite presque invariablement les femmes du côté des «victimes des transitions » et décrit la dégradation de leur condition par la montée de l'instabilité économique, la détérioration des systèmes de protection sociale, la discrimination sur le marché du travail, la diminution de la représentation dans les institutions politiques, la prolifération des discours les traitant en tant qu'objets de consommation sexuelle, la résurgence et le renforcement des conceptions traditionnelles de la division sociale masculin/féminin ${ }^{5}$. Loin de déboucher sur des mouvements de mobilisation des femmes esteuropéennes pour défendre leurs droits, ces évolutions négatives s'accompagneraient d'un désintérêt quasi général pour les idées féministes ${ }^{6}$. Plusieurs raisons sont avancées pour expliquer cet état de fait, qui se réfèrent surtout à l'histoire récente de ces sociétés : réactions de rejet engendrées par le surinvestissement des régimes communistes sur les femmes ; vision de la politique comme «affaire malsaine »; adoption précoce, dans le cadre des politiques égalitaires socialistes, de la majorité des droits faisant l'objet des combats féministes en Occident ${ }^{7}$.

Comme en témoigne le cas roumain, le féminisme des années 1990-2000 - qui s'est toutefois affirmé, dans des conditions que cet article entend examiner - est promu par des personnes provenant des milieux intellectuels. Elles inscrivent la revendication de

\footnotetext{
${ }^{5}$ N. Funk, M. Mueller, 1993 ; B. Einhorn, 1993 ; G. Waylen, 1994 ; Joan W. Scott et alii, 1997 ; T. Renne, 1997 ; S. Gal, G. Kligman, 2000a ; 2000b ; Nouvelles Questions Féministes (« Postcommunisme : genre et Etats en transition»), 23, 2, 2004 ; Transitions ( «'égalité des sexes en Europe centrale et orientale : entre espoir et déconvenues »), XLIV, 1, 2004 ; Signs. Journal of Women in Culture and Society («Eastern Europe »), 29, 3, 2004 ; E. Saurer et alii (éds.), 2006; J. Lukic et alii (éds.), 2006. De rares voix critiquent le stéréotype des «femmes perdantes", le considérant comme le résultat de généralisations insuffisamment ancrées empiriquement (A. Spehar, 2004 ; 2005) ou encore le contredisent de façon ponctuelle par des études de cas centrés sur l'agency féminine (J. Szalai, 1991; 1996 ; A. Bellows, 2001). Enfin, d'autres auteurs mettent en lumière l'instrumentalisation de la condition féminine dans le cadre des discours sur la démocratisation et l'importation a-critique de schémas d'interprétation rattachés à l'idéologie des «femmes victimes des transitions » exercés dans l'analyse d'autres situations de sortie de régimes autoritaires, notamment en Amérique latine (E. S. Werner, 2004 ; V. Whorer, 2004).

${ }^{6}$ Certains auteurs utilisent des formules telles que « antipathie » ou « allergie » au féminisme (B. Einhorn, 1993 ; L. Occhipinti, 1996). Critiquée par la suite avec l'argument que l' "antiféminisme » ne peut être objectivement mesuré, tandis que les indicateurs relatifs à la représentation des femmes dans les institutions politiques ne montrent pas de différences significatives entre les pays ex-socialistes et certains pays occidentaux (Spehar, 2005), l'idée du «rejet» du féminisme est longtemps restée un topos de la littérature consacrée aux transformations postcommunistes en Europe centrale et orientale.

${ }^{7}$ Se reporter aux ouvrages cités en note 5. Voir aussi A. Cerwonka, 2008 et I. Cîrstocea, 2008 pour des aperçus critiques de ces échanges.
} 
l'émancipation féminine dans le cadre d'une philosophie de la «transition » postcommuniste entendue comme moment de modernisation historique et occasion de refonder l'ordre social, voire de questionner les rapports entre les sexes et de repenser la place des femmes dans la société ${ }^{8}$. Regardée dans un premier temps comme une apparition exotique dans le paysage intellectuel et consolidée ensuite par des activités conduites dans l'espace des organisations non gouvernementales $(\mathrm{ONG})$ qui prolifèrent au titre de la démocratisation, la réflexion sur le genre trouve sa place dans le champ académique vers la fin de la décennie 1990 : il s'agit de centres d'études rattachés aux institutions d'enseignement universitaire, pouvant offrir des formations diplômantes, voire même une qualification professionnelle d'«expert pour les questions de genre ». Le premier mastère roumain de ce type fut créé en 1998 à Bucarest et d'autres programmes fonctionnent dans les principales villes universitaires depuis le début des années 2000.

Des centres d'études sur les femmes et/ou sur le genre, créés pendant la décennie 1990, existent dans tous les PECO, de même que dans les pays de l'ex-URSS. Lorsqu'ils ne sont pas rattachés aux universités, ils se présentent comme «indépendants » et il s'agit le plus souvent d'institutions fonctionnant dans le moule des organisations non gouvernementales, dont l'existence est rendue possible par le soutien de divers bailleurs de fonds internationaux : fondations politiques et privées, programmes d'assistance à la démocratisation, organisations et réseaux internationaux d'organisations, chaires occidentales de women's/gender studies'

\footnotetext{
${ }^{8}$ Selon les définitions qu'en donnent ses promotrices roumaines, le féminisme est un «plaidoyer pour les droits des femmes », sachant que celles-ci «sont systématiquement asservies [...], [que] les relations de genre, [qui] ne sont ni naturelles ni immuables [...], sont injustes pour les femmes, [ce qui] impose l'engagement politique pour produire le changement ». C'est à partir de ces idées que les militantes entendent «théoriser, introduire de nouvelles méthodologies scientifiques, rendre à la recherche et à la théorie féministes une autorité épistémique, légitimer scientifiquement les politiques féministes », cf. O. Dragomir, M. Miroiu, 2002, pp. 121 et 123.

${ }^{9}$ La Fondation Soros est un des acteurs les plus investis dans l'entreprise de promotion des droits des femmes et de soutien aux mobilisations féminines dans l'espace ex-soviétique : par le biais de son Women's Program mis en place en 1997, cet organisme y avait consacré avant la fin de l'année 2000 plus de 9,8 million de dollars, alloués sous forme de grants à des associations œuvrant, par ordre d'importance des financements, dans les aires suivantes : «droits humains des femmes», «violence contre les femmes », «genre et éducation » (cf. Open Society Institute, Network Women's Program, Documentation and Evaluation Project, Appendix 3 (Quantitative Data), Open Society Archives, Budapest, fonds HU-OSA 127 1-2-137 (CD-ROM)).
} 
Bien que le degré d'institutionnalisation dans les espaces académiques respectifs et la capacité de recherche de ces centres soient fort inégaux, quelques chiffres peuvent donner une idée des dimensions du phénomène. Selon les données fournies par la Women's/Gender Studies Association of Countries in Transition sur son site destiné à centraliser et à diffuser des informations sur les initiatives de ce type, on enregistrait à la fin de la décennie 1990:3 programmes universitaires et 2 centres indépendants en Bulgarie; un programme universitaire et 2 centres indépendants en Croatie; en Hongrie, 6 programmes universitaires et 3 centres indépendants, auxquels s'ajoute un département «Gender and Culture » dans le cadre de la Central European University à Budapest; en Pologne, 6 programmes universitaires et 2 centres indépendants; en République Serbe, un programme universitaire et 2 centres indépendants; en République Tchèque, 3 programmes universitaires et 2 centres indépendants; en Lituanie 3 programmes universitaires; en Ukraine, 4 programmes universitaires; en Russie, 12 programmes universitaires et 4 centres indépendants ${ }^{10}$. En 1999 , 140 étudiants avaient suivi le programme d'études approfondies sur le genre créé en 1996 à 1’Université de Varsovie ${ }^{11}$.

Comment expliquer le paradoxe sous-entendu par la coexistence du « rejet» du féminisme dans les pays de l'Europe centrale et orientale avec son institutionnalisation rapide dans les espaces scientifiques des mêmes pays? Comment comprendre cette entreprise de création sociale et les mécanismes l'ayant rendue possible ? Comment la lecture du monde social opérée par les chercheuses féministes et par les productions labellisées « études sur le

\footnotetext{
${ }^{10} \mathrm{http}: / /$ www.zenskestudie.edu.yu.wgsact (consulté le 28.12.2004); ces informations furent aussi publiées dans Gender Studies/ Women's Studies Directory, Open Society Institute, 1999. Elles sont toutefois à prendre cum grano salis et à vérifier au cas par cas. Un bilan plus récent des activités circonscrites aux gender studies en Hongrie fait état d'un paysage quelque peu différent : aucune institution d'enseignement supérieur ne propose de diplôme spécialisé, mis à part la Central European University (institution privée fondée sur l'initiative du philanthrope américain G. Soros, longtemps restée isolée du système national d'enseignement, cf. A. Peto (éd.), 2006, p. 79). Egalement, en Bulgarie, en 2007 une seule université était habilitée à encadrer une formation de $3^{\text {e }}$ cycle en gender studies, mais des thématiques spécifiques sont traitées dans plusieurs institutions d'enseignement et de recherche de façon informelle (observation personnelle et entretiens à Sofia, octobre 2007).

${ }^{11}$ M. Fuszara, 2000, p. 1074.
} 
genre » s'inscrit-elle dans le cadre idéologique des «transitions » postcommunistes ? Plus largement, comment les sciences sociales (statut auquel les études féministes prétendent) se situent-elles au sein de l'entreprise de refondation que ces pays traversent depuis le début des années 1990 ? Etant donné l'état d'avancement du présent travail, seules des pistes de réflexion et des hypothèses provisoires peuvent être avancées pour le moment. Elles viennent prolonger une étude consacrée aux luttes pour la définition de l'identité féminine en Roumanie après le tournant politique des années 1990, dont il nous semble utile d'exposer d'abord, brièvement, quelques acquis ${ }^{12}$.

La transition postcommuniste à la lumière des politiques du genre : un aperçu du cas roumain

Afin de déplacer le questionnement et de dépasser les limites habituelles des études «transitologiques », dans notre recherche doctorale menée au sein du Centre de Sociologie Européenne de l'Ecole des Hautes Etudes en Sciences Sociales (EHESS, Paris) nous avons élaboré une étude pluridisciplinaire prenant pour objet les offres et injonctions normatives adressées aux femmes roumaines sur le marché des biens symboliques ouvert par le changement de régime politique dans les années 1990. Cette étude - qui articule l'histoire et la sociologie critique du genre et comporte une variation des niveaux d'analyse - aborde divers pôles de production de l'identité féminine, à deux moments historiques traités de manière comparative: la période d'installation du régime communiste, complétée par un plan rapproché pour la décennie 1960-1970, d'une part, et d'autre part la «transition » des années 1990-2000. Deux axes de réflexion orientent l'ensemble du travail : la construction politique de l'identité féminine lors des moments de refondation de l'ordre social et la mise en

\footnotetext{
${ }^{12}$ I. Cîrstocea, 2004a ; I. Cîrstocea, 2006a.
} 
perspective des discours normatifs et des pratiques sociales. Prenant pour objet d'étude les luttes et conflits symboliques centrés sur la définition de l'identité féminine à un moment de recomposition de l'ordre politique et social, nous nous sommes inspiré des travaux de Joan W. Scott et de Francine Muel-Dreyfus, qui, entre autres, ont défendu l'intérêt et la légitimité d'une approche « genre » en sociologie politique ${ }^{13}$.

La reconstitution historique du processus d'intégration des femmes à la vie politique pendant le régime communiste roumain montre comment le modèle soviétique de l'acteur politique féminin a été adapté en Roumanie dans les années 1950 et érigé en symbole de l'efficacité des politiques socialistes, sans pour autant qu'un bouleversement significatif des représentations traditionnelles des rapports sociaux de sexe ait lieu. Les responsables communistes considéraient les femmes comme des êtres mineurs ${ }^{14}$, tandis que les trajectoires sociales des permanentes du PC, telles que les laissent apparaître les autobiographies d'institution, étaient structurées par la tension entre le devoir militant imposé par la politique d'Etat et le devoir traditionnel envers la famille élargie ${ }^{15}$.

$\mathrm{Si}$, pendant le régime communiste, la transformation de la condition des femmes devait incarner le progrès social, on retrouve une logique similaire dans la théorie sociale de la «transition » (y compris celle produite par des chercheuses féministes), selon laquelle la situation sociale des femmes incarnerait le retard historique de la Roumanie. Alors, on peut constater que les femmes sont à nouveau prises pour cible de stratégies volontaristes d'émancipation et leur condition est instrumentalisée à des fins de progrès social. Parallèlement, la pratique sociale continue de produire des structures de solidarité féminine efficaces dans le quotidien des familles confrontées aux difficultés économiques ${ }^{16}$. Ne s'exprimant pas en tant que telles dans l'espace politique, ces formes de solidarité sont

\footnotetext{
${ }_{13}^{13}$ J. W. Scott, 1988; 1999 ; F. Muel-Dreyfus, 1996.

${ }^{14}$ «Les femmes sont comme des enfants, il faut savoir comment s'y prendre », affirmait-on dans les réunions du bureau politique du PCR, cf. I. Cîrstocea, 2002b, p. 233.

${ }^{15}$ I. Cîrstocea, 2002b ; 2003.

${ }^{16}$ I. Cîrstocea, 2002a ; 2006a; I. Cîrstocea, A. Heemeryck, 2005.
} 
ignorées par les groupes réformateurs qui se servent, dans leur opération de lecture du monde social, de grilles d'interprétation empruntées au répertoire des acteurs internationaux de la démocratisation, ayant un fort contenu normatif ${ }^{17}$. On peut y voir le révélateur d'une déconnexion frappante entre la construction publique des problèmes sur la scène postcommuniste et les expressions non-visibles des pratiques sociales féminines, dont la logique semble perdurer au-delà du changement de régime politique.

Sur la scène postcommuniste qui voit se déployer une vaste production idéologique axée sur la modernisation et le «rattrapage », les définitions normatives de l'identité féminine se multiplient, en même temps que des groupes concurrents agissent dans plusieurs espaces sociaux : le champ politique, la presse et les ONG féminines, l'université. «Moralisés » par les organismes transnationaux (Nations Unies, Banque Mondiale, Commission Européenne) qui ont intensifié dans le courant des années 1990 leurs efforts pour la promotion des droits des femmes, culminant par l'adoption du principe du gender mainstreaming $^{18} »$ lors de la Conférence de Pékin en 1995, les acteurs de l'espace politique roumain postcommuniste s'approprièrent à leur tour la thématique du «genre ». En effet, après une première «transition sans femmes », pendant laquelle aucune préoccupation institutionnelle pour la condition féminine n'a été enregistrée, on peut remarquer un changement d'attitude vers la fin de la décennie 1990. Afin de prouver leur bonne volonté démocratique et recevoir en échange non seulement diverses aides et financements, mais aussi reconnaissance et légitimité sur le plan international, les partis politiques et les institutions d'Etat inscrivent dans leurs

\footnotetext{
${ }^{17}$ La notion désincarnée de « société civile », dont la « faiblesse », voire « l'absence » fut constamment déplorée dans les débats publics des années 1990-2000, en est l'exemple paradigmatique et rend bien compte de la lecture idéologique des évolutions postcommunistes.

${ }^{18}$ Lancé à la IVe Conférence mondiale des femmes organisée par les Nations Unies à Pékin en 1995, le principe du gender mainstreaming désigne la prise en compte du «genre » comme catégorie pertinente dans tous les domaines d'intervention politique. Son adoption couronne deux décennies d'efforts onusiens pour promouvoir et défendre les droits des femmes et il est devenu l'instrument privilégié d'un féminisme étatique et interétatique avec des incidences sur la gestion de la situation des femmes aux niveaux nationaux (voir M. Mintrom, J. True, 2001 ; M. Verloo, 2002 ; S. Roth, 2004 ; 2007 ; S. Jacquot, 2006 (notamment le chapitre I, « Qu'est-ce que le gender mainstreaming? Esquisse d'une genèse », p. 71-133) et les livraisons thématiques Social Politics. International Studies in Gender, State, and Society, 12, 3, 2005; Politique européenne, 20, 2006).
} 
programmes des références à l'« égalité des chances » et au «combat contre la discrimination envers les femmes ${ }^{19}$. Plusieurs départements administratifs ont été créés et des mesures juridiques ont été adoptées pour donner du poids à ces références au niveau pratique, mais le formalisme et le bureaucratisme y sont de rigueur. Par-delà la volonté de faire preuve de progressisme, les questions relatives à la condition sociale et économique des femmes et à leur représentation politique sont, à présent comme dans le passé, traitées comme s'il s'agissait d'un registre mineur.

Considéré à travers les jeux politiques autour du genre, le postulat du «changement » postcommuniste apparaît donc nuancé, si bien que, s'il est possible de parler, dans les années 1990-2000, d'une mutation des politiques en rapport avec la «question femme », elle se situe, mis à part le renouvellement et la multiplication des acteurs, surtout au niveau des formules et du modèle plutôt qu'à celui de la logique et de la praxis. Dans les départements administratifs bâtis sur mesure et baptisés dans le jargon international «mécanismes nationaux pour la promotion des femmes », ce n'est plus «le modèle soviétique » qui fonctionne comme référence immédiate, mais les conventions de l'ONU et l' «acquis communautaire », devenus désormais les repères sine qua non des acteurs nationaux engagés dans le traitement politique de la condition féminine ${ }^{20}$.

La nouvelle doxa relative aux femmes ne passe pas exclusivement par les relais des partis politiques et des institutions d'Etat, mais elle est véhiculée aussi par des organisations non gouvernementales. Produits typiques des situations de décolonisation, dont les sociétés postcommunistes se rapprochent selon certains auteurs, elles prolifèrent en Europe centrale et orientale sous l'impulsion consistante des organismes transnationaux qui y voient des agents-

\footnotetext{
${ }^{19}$ E. Zamfir, 1999 ; V. Stoica, 2003 ; G. Ghebrea et alii, 2005a ; 2005b ; I. Borza et alii, 2006. L'adoption de politiques pour l'égalité de genre traduit la même volonté d'obtenir de la légitimité auprès des acteurs internationaux (notamment l'Union Européenne) dans bien d'autres pays est-européens : comme l'affirme une spécialiste de la question, c'est «une des choses qui peuvent être faites pour montrer l'appartenance à l'Occident, à l'Europe » (M. Verloo, 2002, pp. 112-113). Selon d'autres auteurs, y compris les gender studies furent adoptées par certaines institutions est-européennes d'enseignement supérieur afin de traduire une intention de modernisation et d'occidentalisation (S. Zimmermann, 2007).

${ }^{20}$ I. Cîrstocea, 2006a.
} 
clé de la démocratisation, voire l'incarnation même de la «société civile ${ }^{21}$. En Roumanie, au début des années 2000, plusieurs dizaines d'organisations se consacraient aux activités en faveur des femmes, plus de $85 \%$ de leurs programmes dépendant des bailleurs de fonds internationaux et leurs statuts reprenant parfois tels quels les objectifs de l'intervention des agences transnationales d'aide à la démocratisation ${ }^{22}$. Un cas extrême qui mérite d'être évoqué pour l'anecdote montre que de telles institutions peuvent être bâties sur mesure afin de rentrer parmi les clients potentiels des programmes internationaux : après avoir appris par des voies officieuses que l'Union Européenne allait financer des programmes de soutien aux femmes, une femme-entrepreneur roumaine monta une fondation profilée sur ce genre d'activités ${ }^{23}$.

Se distinguant dans le paysage associatif roumain des années 1990 comme l'unique organisation qui affiche une identité féministe dans son intitulé, la Société d'analyses féministes AnA de Bucarest, créée en 1993, fut le principal relais de diffusion de la réflexion sur le genre en Roumanie ${ }^{24}$. Les propriétés sociales de son personnel, qui se trouve aussi à l'origine de la création du premier module de gender studies dans l'enseignement supérieur de ce pays, montrent que le féminisme des années 1990 n'est pas l'œuvre d'ex-militantes communistes pour les droits des femmes. Bien au contraire, les personnes engagées dans cette entreprise sont de jeunes intellectuelles précédemment marginales dans l'espace académique, qui y font leur entrée en tant que porteuses d'un nouveau domaine de production de

\footnotetext{
${ }^{21}$ Pour des approches critiques des ONG en tant qu' « outils de la globalisation», voir par exemple B. Hours, M. Selim, 2003 ; Journal des anthropologues («Les ONG : médiations politiques et globalisation »), 94-95, 2003. Sur l'action des organismes transnationaux en Europe de l'est, voir J. Wedel, 1998 ; N. Ragaru, 2000-2001 ; G. Pirotte, 2003 ; T. Carothers, 2004. Spécifiquement sur le thème de l'« ONGization » du féminisme, voir S. Lang, 1997 ; L. Grunberg, 2000b ; J. True, 2003 ; M. Misiorowska, 2004 ; A. Sloat, 2005 ; K. Ghodsee, 2004 ; 2006 ; A. Bagic, 2006 ; S. Roth, 2007. Voir aussi N. Funk, 2006, qui montre que, malgré les innombrables critiques de tous les horizons idéologiques qui ont pu leur être adressées, ces institutions ont quand même rendu possible des activités autrement difficilement envisageables, y compris la recherche sur le genre.

${ }^{22}$ L. Grünberg, 2000a ; I. Cîrstocea, 2006a. Pour des chiffres similaires sur le terrain ex-yougoslave, voir A. Bagic, 2006, p. 161 : «peut-être plus de 80-90\% d'entre elles n'auraient pas existé en absence du support financier extérieur, ou bien leur développement aurait été très différent » (la traduction nous appartient).

${ }^{23}$ G. Pirotte, 2003, p. 176. Une histoire subjective d'une ONG roumaine de droits des femmes publiée récemment fait également état de nombreuses situations d'ajustement entre «offre «et «demande » en matière de promotion de la démocratie, cf. L. Grünberg, 2008 .

${ }^{24}$ I. Cîrstocea 2004b; I. Cîrstocea 2006a, notamment le chapitre 2.
} 
connaissances. En effet, après une initiation théorique au «féminisme académique » dans des universités occidentales, l'engagement public en faveur de la cause des femmes développé dans les milieux associatifs (ONG) leur offre les moyens d'une reconversion professionnelle et contribue à leur consécration dans les espaces scientifique et politique au niveau national, qui finissent par reconnaître leur «expertise » sur le genre. Une importante mobilisation de ressources individuelles et collectives acquises pendant le régime antérieur transparaît dans la constitution du groupe militant, même si, au niveau de la représentation du passé, la volonté affirmée de ces actrices est de rompre avec l'héritage socialiste, présenté à travers des images diabolisées, et de renouer avec le militantisme féministe des débuts du XXe siècle, découvert sur le tard et érigé en source de légitimité ${ }^{25}$.

Illustrant la complexité des enjeux dont relève la «question femme » dans sa version actuelle, le féminisme roumain postcommuniste est un courant intellectuel par excellence ambigu, situé au croisement de la critique épistémologique et de l'innovation en sciences sociales avec l'expertise au service des réformes politiques et la philosophie sociale propre aux intellectuels engagés dans la modernisation du pays. Par ailleurs, le cas roumain illustre bien le caractère multipositionnel ${ }^{26}$ du féminisme au tournant du XXIe siècle : les acteurs militants investissent simultanément des arènes contrastées et utilisent une grande diversité de stratégies et ressources pour faire entendre leurs revendications, malgré l'essoufflement apparent des mouvements sociaux des femmes au sens classique, consacré par les mobilisations occidentales des années $1970^{27}$.

\footnotetext{
${ }^{25}$ I. Cîrstocea, 2006 b.

${ }^{26}$ L. Boltanski, 1973.

${ }^{27}$ M. M. Ferree, 2006.
} 


\section{Construction nationale et internationale du genre après le communisme :}

\section{quelques pistes de réflexion}

La problématique de l'instrumentalisation politique des femmes au sein de la construction idéologique des «transitions » postcommunistes, dont les sciences sociales sont des acteurs à part entière, mérite d'être approfondie et étudiée dans une perspective comparative à partir de plusieurs cas nationaux. Il est utile d'interroger dans chaque cas les rapports entre les revendications féministes des années 1990-2000 et les anciennes préoccupations relatives à la condition des femmes, formulées de diverses façons par les régimes communistes : les études sur le genre offrent-elles des possibilités de reconversion aux préoccupations, aux acteurs et aux réseaux constitués et affirmés dans le cadre des politiques socialistes d'émancipation des femmes ? Peut-on observer dans la rhétorique des nouveaux agents une réappropriation de l'argumentaire élaboré pendant les régimes antérieurs ou de celui appartenant aux militantes féministes des débuts du XXe siècle? Avec quel succès ? Plus largement, comment l'histoire est-elle utilisée sur le nouveau marché des biens symboliques ouvert par le changement de régime politique, d'une part, et par l'internationalisation bureaucratique et scientifique du féminisme, d'autre part ?

Au premier abord, plusieurs éléments différencient les situations nationales, à savoir : l'existence ou l'absence de mobilisation civique des femmes hors de l'université, des ONG et du «féminisme d'Etat» affirmé au titre de l'adoption des normes relatives aux «droits humains des femmes » et au «gender mainstreaming ${ }^{28} ;$ l'existence ou l'absence de formes de participation de groupes structurés sur le critère de l'identité de genre à la compétition politique proprement dite (partis ou alliances féminins inscrits sur les listes électorales, par exemple $)^{29}$; l'existence ou l'absence de préoccupations pour le «féminisme académique » et

\footnotetext{
${ }^{28}$ Dans certains pays (Pologne, Allemagne, Slovénie), des mobilisations féminines aux dimensions et effets variables ont été provoquées par la mise en question du droit à l'avortement au début des années 1990.

${ }^{29}$ En Russie, des groupements de femmes se sont présentés sur des listes séparées aux élections pour la Douma en 1993 (A. Posadskaya-Vanderbeck, 1997). Des «partis des femmes » ont sporadiquement été enregistrés en
} 
les women's/gender studies antérieures à l'effondrement des régimes communistes. Plusieurs exemples montrent l'existence de différences et de contrastes qui pourraient être pris en compte pour construire une éventuelle typologie. A Moscou, l'un des centres d'études féministes les plus dynamiques a été fondé sur une initiative d'Etat, dont il tire une partie de ses financements. A Prague, le Centre d'études sur le genre a d'abord fonctionné sous la forme d'une association professionnelle et les enseignements féministes ont pénétré plus tard dans l'espace universitaire, en l'absence de revendications féministes de la «base » populaire, mais bénéficiant, par contre, de l'impulsion et du soutien de divers groupes militants et institutions occidentaux ${ }^{30}$. De son côté, le cas ex-Yougoslave représente encore une autre situation : plusieurs congrès en sciences sociales centrés sur des thèmes féministes y ont été organisés à partir de la fin des années 1970-1980, tandis que des organisations féminines centrées sur des questions pratiques (lignes téléphoniques d'urgence, refuges pour femmes et enfants victimes de violences et d'abus) existaient à la fin des années 1980 dans la plupart des villes-capitales. Une partie des ressources symboliques constituées à ce moment-là furent par la suite investies dans des mobilisations contre les guerres interethniques, ainsi que dans les centres de recherches et d'enseignement sur les femmes et le genre, dont le plus ancien fonctionne à Belgrade depuis 1992. A remarquer aussi que les militantes ex-yougoslaves n'opèrent pas le même découpage chronologique entre l' «avant» et 1 ' «après » régime socialiste mais identifient une ligne de clivage dans leur expérience sociale et politique en fonction des guerres et du démantèlement de la fédération ${ }^{31}$.

Ces jalons préliminaires étant posés, on peut procéder à la formulation de quelques directions de recherche encore rarement abordées par les travaux d'histoire du féminisme et

Hongrie (A. Peto, 2001), en République Tchèque (B. Einhorn, 1993), en Serbie (V. Litrichin, L. Mladenovic, 1997).

${ }^{30} \mathrm{~J}$. True, 2003.

${ }^{31}$ A. Bagic, 2006 ; Z. Lorand, 2007; C. Bonfiglioli, 2008. 
les études de sociologie des espaces scientifiques, qui négligent dans une large mesure les pays d'Europe centrale et orientale.

\section{Les études féministes : une nouvelle discipline académique?}

Quels agents se trouvent à l'origine de l'institutionnalisation des women's/gender/feminist studies dans les pays ex-communistes? Quelles sont les luttes de classement auxquelles elles donnent lieu dans les champs scientifiques nationaux ? En quoi leur apparition contribue-t-elle à modifier les espaces professionnels académiques de ces pays ? Produisent-elles des mutations au niveau des hiérarchies des «establishments» scientifiques?

Pour répondre à ces questions, le paradigme de l'histoire des sciences sociales semble le plus approprié, vu sa vocation à prendre pour objet d'étude les instances propres aux espaces scientifiques, leurs agents, l'état des problématiques, la hiérarchie des objets et des outils intellectuels disponibles à un moment donné ${ }^{32}$. Les conditions politiques et socioéconomiques de fonctionnement des espaces académiques d'accueil, avec leurs luttes et conflits, définissent la réception des problématiques, des concepts, des méthodes et des pratiques de recherche propres aux études féministes comme aux toutes autres nouvelles préoccupations scientifiques. Abordant dans cette perspective les espaces scientifiques d'Europe centrale et orientale, on peut tester les acquis théoriques et méthodologiques de la sociologie critique sur un terrain nouveau et élargir ses interrogations et réflexions portant sur les pratiques professionnelles des spécialistes en sciences sociales. Au-delà de son intérêt purement intellectuel, cette démarche est une entreprise nécessaire à un moment où les sciences sociales se recomposent dans cette région du monde, au terme d'une longue période

\footnotetext{
${ }^{32}$ Pour la définition de l'histoire des sciences sociales et du programme de recherche correspondant, nous nous référons ponctuellement à J. Heilbron et alii, 2004. La toile de fond de la réflexion est fournie par le programme de sociologie des sciences élaboré par Pierre Bourdieu (P. Bourdieu, 1984 ; 1997 ; 2002a ; 2002b).
} 
d'annexion, voire d'assujettissement idéologique ${ }^{33}$. L'intérêt d'étudier les recompositions des sciences sociales en Europe centrale avec les outils de la sociologie critique a été souligné depuis longtemps ${ }^{34}$. Quelques travaux mettent en lumière la contribution des acteurs internationaux, tels que les fondations privées et politiques occidentales, au renouvellement et à la diversification des élites intellectuelles et politiques est-européennes après le changement de régimes, à travers des programmes de bourses et des « centres d'excellence ». Ces mêmes études abordent aussi dans une perspective critique les processus sociaux et politiques de construction des paradigmes conceptuels mobilisés pour étudier les phénomènes postcommunistes $^{35}$.

Pour revenir aux gender studies, on sait que dans leur espace originaire (occidental, notamment nord-américain), ce sont les mobilisations féministes des années 1960-1970 qui ont progressivement entraîné des questionnements critiques sur les sciences sociales conduisant à l'élaboration de paradigmes théoriques à même d'englober les rapports sociaux de sexe. Des études comparatives récentes portant sur plusieurs pays d'Europe occidentale montrent des similarités entre les divers cas nationaux, en dépit de certaines différences quant aux temporalités de l'institutionnalisation et au degré de reconnaissance par les communautés académiques. En effet, les études féministes y sont apparues dans la continuité des mouvements de libération des femmes, sous la forme de recherches d'abord, puis d'enseignements; quelques phases distinctes ont pu être observées de manière générale, à savoir : «militante », «de l'institutionnalisation », « de la professionnalisation », « de l'autonomie $»^{36}$.

\footnotetext{
${ }^{33}$ Revue d'histoire des sciences humaines («Quelle sociologie derrière le 'rideau de fer’ ? 1950-1989»), 14, 2007 ; Journal des anthropologues («Parcours de l'ethnologie dans le monde postsoviétique »), 87, 2001 pour des réflexions utiles à ce propos.

${ }^{34}$ R-M Lagrave, 1998.

${ }_{35}^{35}$ N. Guilhot, $2001 ; 2004 ; 2005 ; 2007$; M. D. Gheorghiu 2004.

${ }^{36}$ M. Andriocci et alii, 2003 ; G. Griffin, 2005.
} 
Au contraire, l'émergence et l'institutionnalisation rapide des études féministes dans les PECO relèvent d'initiatives limitées à des groupements intellectuels déconnectés de la majorité de la population féminine. Ces acteurs s'inscrivent dans des logiques internationales de communication scientifique et se font porteurs de problématiques relevant d'un répertoire théorique élaboré sur la base d'expériences sociales éloignées, devenu déjà, au moment de sa diffusion vers l'Europe de l'est, partie d'un référentiel érigé en norme internationale et promu par des moyens bureaucratiques. Si bien qu'une chercheuse croate a pu décrire le «pattern» est-européen comme une « imposition » du genre, à travers des mobilisations transnationales, par des autorités scientifiques, des programmes d'échanges et des initiatives occidentales ${ }^{37}$.

L'hypothèse que nous avançons à la lumière de nos travaux antérieurs, après avoir balisé, ne serait ce que superficiellement, le champ des études féministes dans les pays excommunistes, soutient que l'émergence de ces études dans les PECO relève dans une large mesure d'un processus d'internalisation des normes transnationales de promotion des droits des femmes. A part sa dimension proprement bureaucratique, consistant à faire adopter des mesures juridiques et à créer des départements administratifs devant veiller à la non discrimination envers les femmes, ce processus revêt aussi une dimension touchant aux sciences sociales qui se voient enrôlées pour combattre les stéréotypes et produire les «bonnes » représentations des relations masculin/féminin et des rôles sociaux des sexes ${ }^{38}$. Ayant acquis récemment droit de cité dans les universités est-européennes, les études sur le genre s'inscrivent en effet simultanément à plusieurs niveaux, qui s'entrecroisent et s'influencent réciproquement (militant et savant, national et international), et dans plusieurs

\footnotetext{
${ }_{38}^{37}$ B. Kasic, 2004a, p. 31.

${ }^{38}$ Le texte de la Convention des femmes (CEDAW), par exemple, recommande que soient « révisés les livres, les programmes scolaires et les méthodes pédagogiques afin d'en éliminer toute conception stéréotypée des rôles des hommes et des femmes » (http://www.un.org/womenwatch/daw/cedaw/fconvention.htm). D'autres manifestes définissent l'éducation sur le genre comme un «entraînement pour la démocratie » et les women's/gender studies comme un «facteur majeur dans la construction de la cohésion sociale et du respect démocratique pour les droits humains » (R. Braidotti, 2004, p. 10), ou bien comme un «outil servant à la rééducation culturelle pour que soient abandonnées les pratiques discriminatoires » (R. Braidotti, 2002, p. 303, la traduction nous appartient).
} 
espaces sociaux (scientifique, associatif et politique). Chacun de ces espaces est caractérisé par ses propres hiérarchies et luttes de classement, mais leurs frontières sont perméables, comme en témoignent les investissements multiples des agents sociaux engagés dans les gender studies : en Roumanie, par exemple, les pionnières du domaine militent dans des ONG, donnent des enseignements sur le genre et occupent des positions d'experts contribuant à l'élaboration de politiques publiques ciblant les femmes.

L'analyse sociologique des processus d'inscription de ces études dans les espaces universitaires nationaux des PECO ne peut pas faire l'économie de la reconstitution des luttes de classement propres à ces espaces et des ressources mobilisées par les agents qui investissent ce nouveau domaine. Il s'agit de comprendre en quoi les milieux scientifiques ont été modifiés par l'apparition de ce nouveau champ de recherche et d'enseignement. Le cas roumain montre que les chercheuses féministes font valoir dans l'espace académique national des formes de capital exogènes ; associations, institutions politiques, institutions scientifiques occidentales font figure d'instances de légitimation en concurrence avec les instances propres à l'espace universitaire national, lequel accueille, avec bien des réserves, les thématiques relatives au genre et les nouveaux spécialistes qui les portent. Toutefois, étant en étroite correspondance avec des thématiques privilégiées par les programmes de démocratisation et les réformes institutionnelles menées d'un côté au nom du combat international contre la discrimination à l'égard des femmes, de l'autre au nom de la modernisation du système universitaire, la nouvelle forme d'enseignement trouve sa place dans l'espace professionnel national et contribue à le transformer. On voit donc que les dynamiques transnationales catalysent les luttes de classement et les conflits de légitimité dans les espaces académiques nationaux des PECO; plus spécifiquement, on peut considérer qu'elles impulsent la structuration des champs académiques professionnels nationaux et permettent l'affirmation de nouveaux agents. 
Lorsque l'on se penche sur la dimension proprement politique de l'institutionnalisation des études sur le genre en Europe, d'autres rapports, complexes, apparaissent, au niveau national et au niveau international, voire transnational. Comme en témoignent les agents impliqués dans ces processus, les études sur le genre se voient confier le rôle de projet intellectuel et politique fédérateur dans le cadre de la construction européenne. Elles sont imaginées comme un relais possible pour l'expression d'une «identité européenne » et comme base d'une communication qui dépasse les frontières nationales. Cette représentation apparaît aussi dans l'intention, affirmée par certains agents-clé de l'institutionnalisation de ce domaine, de construire une «dimension européenne » des études sur le genre, qui soit ancrée dans des problématiques spécifiquement «européennes », éventuellement différentes de la tradition nord-américaine des «gender studies». Le support européen pour l'institutionnalisation des études sur le genre, dans le cadre de la création de l'Aire scientifique européenne et des réformes universitaires entamées vers la fin des années 1990 avec les accords de Bologne, se traduit par la promotion des échanges interuniversitaires, la création de réseaux multinationaux et interdisciplinaires axés sur le développement de programmes communs d'enseignement et de recherche, ainsi que, depuis peu, par la mise en place de diplômes de mastère conjoints impliquant plusieurs universités partenaires ${ }^{39}$. Il est sans doute trop tôt pour tirer une conclusion sur les résultats de ce processus d'ingénierie intellectuelle et institutionnelle. Notons toutefois que, malgré l'optimisme des rapports réguliers, les opinions des acteurs et bénéficiaires sont assez mitigées, nombre d'entre eux déplorant certains effets pervers des réformes «LMD» qui semblent renforcer les frontières disciplinaires et, du coup, ne pas permettre l'affirmation plénière des gender studies, par

\footnotetext{
${ }^{39}$ ENWS, 1993 ; R. Braidotti et alii, 1995 ; R. Braidotti, G. Griffin, 2002 ; E. Magyari-Vincze, 2002 ; Griffin, 2005. Voir aussi la série intitulée The Making European of Women's Studies éditée depuis 2000 par le réseau international ATHENA de promotion des études féministes européennes, basé à l'Université d'Utrecht (8 volumes parus à ce jour).
} 
excellence interdisciplinaires ${ }^{40}$. Retenons pour l'heure que l'émergence des études féministes constitue aussi un découpage analytique pouvant servir à comprendre la construction de 1' «Europe » entendue comme projet transnational, avec des logiques propres, articulées sur des logiques nationales et internationales à travers une «diversité de transactions, négociations et réinterprétations qui se jouent sur différentes scènes autour d'une grande variété d'objets $»^{41}$.

Malgré la prolifération des publications consacrées au développement des études féministes en Europe centrale et orientale, il faut souligner le fait que la réflexion systématique et relationnelle sur leur insertion dans les espaces universitaires d'accueil est à peine entamée ${ }^{42}$. Les travaux disponibles appartiennent, pour la plupart, aux acteurs même de l'institutionnalisation ; ce sont le plus souvent des plaidoyers pro domo, ou bien des comptesrendus détaillés des acquis, conçus en termes de nombre de centres, de formations, de publications, d'étudiants. Pourtant, il ne faut pas ignorer l'existence d'une réflexion développée par les professionnelles des études féministes elles-mêmes au sujet du statut et du rôle du domaine de connaissances qu'elles sont en train de bâtir. Les universitaires et chercheuses spécialistes des «gender studies » dépensent beaucoup d'énergie autour d'un débat classique de la recherche féministe, à savoir le dilemme «autonomie vs. intégration », argumentant pour ou contre la nécessité de constituer une discipline en soi, ou bien la possibilité d'adresser un questionnement transversal aux disciplines existantes. La plupart des « voix » optent pour le « oui » de l'autonomie et l'institutionnalisation qui la renforce, l'une et l'autre pouvant être mesurées selon certains critères: le nombre de postes et de chaires universitaires, le nombre de facultés ou de départements spécialisés, la qualité des

\footnotetext{
${ }^{40}$ Cf. observations et entretiens personnels à Budapest (mai 2007, avril 2008) et à Sofia (octobre, 2007). Des réserves du même ordre s'expriment aussi dans un volume consacré au développement des gender studies en Hongrie (A. Peto (éd.), 2006, 81).

${ }^{41}$ M. Werner, B. Zimmermann, 2004, p. 28.

${ }^{42}$ S. Zimmermann, 2007 est un exemple.
} 
professionnels impliqués, la capacité d'accorder des diplômes, le montant et le type de budgets, la capacité de recherche, la reconnaissance par les corps dirigeants de l'enseignement universitaire $^{43}$. Des colloques et des conférences sont donc organisés pour débattre de l'utilité de la définition d'un «canon » des études féministes, des publications sont diffusées qui répertorient et recommandent les «bonnes pratiques » dans l'enseignement et la recherche spécialisés en études «genre ${ }^{44}$. Toutefois, le risque demeure de sacrifier la portée critique et la vocation déstabilisatrice originaire des questionnements féministes dans les sciences sociales. C'est pourquoi d'autres spécialistes, qui reconnaissent par ailleurs les avantages de l'institutionnalisation, à savoir l'autonomie et les ressources dues à l'affirmation en tant que discipline scientifique, plaident pour que les études féministes soient considérées plutôt comme une «post-discipline », en raison de leur capacité particulière à défier les frontières, les canons, les méthodes classiques et les autorités scientifiques ${ }^{45}$.

Mettant en avant la vocation des gender studies à produire un savoir utile à des fins de transformation sociale, les porte-paroles de ces études ne questionnent pas la relation de leur domaine avec les instances extérieures à l'espace académique : il s'agit notamment des rapports avec divers agents de l'espace politique, qui peuvent intervenir dans la définition des thématiques et des problématiques à aborder sous l'angle du genre. Ces rapports sont transparents dans les discours des bureaucrates qui impulsent et soutiennent la mise en place des études féministes, en définissent le rôle et l'orientation pragmatique et évoquent le soidisant «triangle de velours » réunissant les organisations féminines (voire le mouvement féministe), les policy makers et les universitaires, dont seule la convergence est susceptible de

\footnotetext{
${ }^{43}$ G. Griffin, 2004, p. 205. D'autres admettent que l'existence d'un corpus établi de théories et de publications suffit pour que les études féministes/sur le genre soient considérées comme une discipline à part entière (B. Einhorn, 2002, p. 83).

${ }^{44}$ L. Grünberg, 2001 en est un exemple entre autres. Voir aussi Braidotti, Griffin, 2002, présenté comme le premier manuel européen de women's studies.

${ }^{45}$ N. Lykke, 2004.
} 
conduire à une véritable émancipation des femmes et à l'égalité des chances ${ }^{46}$. Cela suggère une particularité de la nouvelle «discipline» qui reste à vérifier de façon systématique : malgré son aspiration à une identité scientifique, elle est « hétéronome » par excellence, vu sa dépendance originaire par rapport à des instances de légitimation extra-académiques.

\section{Logiques pratiques du féminisme international}

En Europe centrale et orientale, les gender studies ont une vocation transnationale constitutive, confortée par plusieurs arguments dont nous évoquons brièvement quelques uns. L'impulsion donnée par les milieux occidentaux, notamment nord-américains, à l'insertion des préoccupations féministes, unanimement reconnue dans les pays est-européens, transparaît dans de nombreuses publications qui témoignent du fait que certaines organisations sont créées à l'initiative de militantes ou d'associations occidentales, voire de réseaux internationaux d'ONG ${ }^{47}$. Notons aussi que ce sont les exigences des organismes internationaux (Programme des Nations Unies pour le Développement, Union Européenne, Banque Mondiale) qui ont suscité la collecte de données quantitatives et la production de statistiques «sensibles au genre ${ }^{48}$ » dans cette région du monde. A un autre niveau, des connexions s'établissent entre les départements spécialisés de gender studies, qui développent des projets de recherche en réseaux et utilisent l'anglais comme lingua franca dans les échanges scientifiques, certains d'entre eux allant jusqu'à proposer des enseignements d'« anglais pour les études de genre ${ }^{49}$. L'usage des nouvelles technologies de l'information

\footnotetext{
${ }^{46}$ R. Braidotti et alii, 2004, pp. 141 et 144.

${ }^{47}$ B. Einhorn, 1993 ; L. Busheikin, 1997 ; A. Spehar, 2004; B. Kasic, 2004a; A.Snitow,1995 ; 2006 ; A. Bagic, 2006. Selon S. Zimmermann, selon la source de financement des travaux sur le genre, on peut distinguer entre «le temps des Américaines » (début des années 1990) et celui « de l'Europe » (après le tournant des années 2000), cf. S. Zimmermann, 2007.

${ }^{48}$ En Roumanie, l'Institut National des Statistiques de Bucarest mène depuis 1998, avec l'appui du PNUD, un projet intitulé « Engendering Statistics ».

49 The Academic Year 2001/02 Annual Report of Belgrade Women's Studies Center, www.zenskestudie.edu.yu (consulté le 28.12.2004).
} 
et de la communication que font les chercheuses féministes s'inscrit dans la même logique ; en témoigne la prolifération des bibliothèques virtuelles d'études féministes, des bases de données en ligne, des forums de discussion. Les « conversions » individuelles au féminisme et les modes de recrutement des scientifiques militantes relèvent souvent de processus de circulation internationale. Dans le cas roumain, l'exemple paradigmatique est celui de la principale porte-parole du «féminisme académique », une professeure de sciences sociales dans le secondaire qui entra en contact avec les théories politiques et la philosophie féministes américaines à l'occasion d'échanges avec des collègues occidentaux et d'un voyage professionnel entrepris au début des années $1990^{50}$. Enfin, la volonté de «se connecter à l'histoire du mouvement international des femmes» et de rappeler l'expérience de la solidarité féminine internationale transparaît dans le choix du titre d'une publication éditée par un centre féministe de documentation en Croatie dans les années 1990, qui reprend le nom d'un groupe pour la libération des femmes actif à Boston dans les années 1970 (« Bread and $\left.\operatorname{Roses}^{51} »\right)$.

Sur la base de ces observations, on peut imaginer une réflexion comparative sur l'émergence de ce nouveau domaine académique, afin non seulement d'éclairer certains «impensés » des travaux d'histoire du féminisme mais aussi de mieux comprendre les ressorts eux-mêmes du soi-disant féminisme «de la globalisation », qui n’a que très peu été interrogé sous l'angle des interactions entre les agents sociaux qui le rendent possible. Comment donc les mobilisations féminines transnationales fonctionnent-elles pratiquement? Quels sont les rapports sociaux qui s'établissent à travers la communication entre les agences transnationales et les organisations nationales de femmes, entre les professionnelles occidentales des études sur le genre et leurs pairs (souvent disciples) est-européennes?

\footnotetext{
${ }^{50}$ I. Cîrstocea 2004b; 2006a.

${ }^{51}$ A. Bagic, 1997, p. 201. Voir I. Cîrstocea, 2008 sur d'autres stratégies d'inscription du féminisme est-européen dans l'histoire internationale des mouvements des femmes, notamment l'adoption de la chronologie consacrée des « vagues ».
} 
Nous allons à nouveau faire référence à Pierre Bourdieu et à sa sociologie de la circulation internationale des biens symboliques pour souligner que la vie intellectuelle n'est pas spontanément internationale et que tout transfert est une opération sociale de sélection, de marquage, de lecture, dont il est important de connaître les conditions sociales de possibilité et de mesurer les effets cognitifs et politiques $^{52}$. Cette connaissance est d'autant plus nécessaire qu'il s'agit d'idées, théories, concepts féministes postmodernes et poststructuralistes définis par la qualité de «voyager ${ }^{53} »$, de traverser non seulement les disciplines académiques, mais aussi les espaces sociaux, culturels et géographiques. La notion de «genre » en est l'exemple par excellence et l'Europe de l'est - en tant qu'espace où elle fut adaptée récemment et en déconnexion des débats auxquels elle doit sa genèse ${ }^{54}$ - nous apparaît comme le terrain parfait pour étudier les effets politiques et épistémologiques des phénomènes de circulation internationale des idées.

Les interactions entre les féministes des PECO et leurs interlocutrices occidentales n’ont pas été systématiquement questionnées sous cet angle, bien que, dès le début des années 1990, de nombreux articles et ouvrages produits d'un côté et de l'autre de l'Atlantique aient abordé la question de la possibilité d'importer et adapter en Europe de l'est des problématiques, concepts et paradigmes explicatifs élaborés à l‘Ouest. Remarquablement vifs pendant la décennie 1990, les échanges au sujet du féminisme, connus aussi comme le «malentendu Est-Ouest ${ }^{55} »$, ont conduit à une intéressante révision conceptuelle et à une nouvelle critique du supposé universalisme des luttes féminines. En effet, ils ont consacré dans le répertoire théorique du féminisme international un nouveau clivage, venu s'ajouter à ceux de race, classe et orientation sexuelle affirmés depuis longtemps par des courants

\footnotetext{
${ }^{52}$ P. Bourdieu, 2002b. Voir aussi J. Heilbron et alii, 2008.

53 « Travelling concepts » (M. Bal, 2002); «travelling theory » (A. Cerwonka, 2008); "genre nomade" (Sociétés contemporaines, 2008, 3, 71).

${ }^{54}$ J. W.Scott, 2004; J. Butler, E. Fassin, J. W. Scott, 2007.

${ }^{55}$ N. Funk, 1993; 2007 ; L. Busheikin, 1997; A; Snitow, 1995; 2006; M. Frunza, E. Vacarescu, $2004 ;$ R. Muharska, 2005 ; K. Slavova, 2006 ; A. Cerwonka, 2008.
} 
contestataires du féminisme «mainstream » (occidental, blanc, de classe moyenne), portés par des groupes issus de cultures postcoloniales ou par des groupes minoritaires (femmes noires, lesbiennes). Il s'agit de la divergence due à la socialisation sous des régimes socialistes, qui conduit à une interprétation différente des principaux thèmes de mobilisation des femmes occidentales et qui explique en partie la réception problématique du répertoire théorique et pratique de ces dernières en Europe de l'est pendant les années $1990^{56}$.

Si la plupart des études récentes consacrées aux mouvements des femmes soulignent la dimension transnationale du féminisme contemporain, l'étude sociologique de sa production, de sa circulation et de sa réception reste une entreprise difficile. Les travaux consacrés aux échanges Est-Ouest avancent quelques propositions théoriques intéressantes mais rares sont les études de cas concrets, portant sur des terrains précis. Au titre des suggestions heuristiques qui visent à déplacer l'accent analytique du «clivage» Est-Ouest vers la dynamique des processus transnationaux, nous pouvons citer deux articles qui, tout en critiquant la réification des frontières géographiques et l'essentialisation des notions de « Est » et « Ouest », insistent sur l'utilité d'étudier la diffusion internationale des idées féministes, à travers, respectivement, les concepts de «réverbérations féministes ${ }^{57}$ » et de « $\operatorname{transculturation}^{58}$ ». Quant aux études plus appliquées consacrées à la circulation des idées féministes vers l'Europe de l'est, on peut se référer à une recherche centrée sur les traductions de textes féministes occidentaux en Hongrie dans les années 1990-2000 ${ }^{59}$, ainsi qu'à une analyse entreprise sur la base d'observations de séminaires de formation des militantes organisés par des professeures américaines auprès d'associations de femmes russes ${ }^{60}$. L'étude des processus de transferts idéologiques et des rapports sociaux qui s'établissent à travers les interactions

\footnotetext{
${ }^{56}$ M.M. Ferree 1995 est une des plus claires synthèses sur ce point.

${ }^{57}$ J.W. Scott, 2004.

${ }^{58}$ A. Cerwonka 2008.

${ }^{59}$ S. Gal, 2003.

${ }^{60}$ M. M. Ferree et alii, 2001. Voir aussi A. Hrycak, 2002.
} 
montre que la transmission n'est ni unilinéaire ni dépourvue de tensions (rapports hiérarchiques, concurrence pour les ressources, etc.) ${ }^{61}$.

Les observations que nous avons pu entreprendre jusqu'à présent soutiennent l'intérêt d'élaborer une ethnographie fine des contacts entre groupes féministes en Europe de l'est et d'une observation, poursuivie sur le long terme, des opérations de sélection des problématiques et des thématiques érigées en sujets d'études ou de débats théoriques. Afin d'illustrer le malaise de l' «acculturation» subie par certaines actrices est-européennes engagées dans les échanges internationaux, qui font état de phénomènes d'imposition de problématiques restant à analyser de façon systématique, citons les propos d'une chercheuse roumaine. Tout en faisant des efforts pour s'approprier des outils conceptuels acquis par la fréquentation des milieux occidentaux, elle exprime sans équivoque la difficulté de rendre intelligible à travers leur prisme la réalité sociale immédiate : «Loin d'être des victimes de la transition, les femmes en sont les managers ${ }^{62}$; ou bien : «Nous [les intellectuelles féministes] lançons des stratégies pour l'égalité des chances, nous proposons des changements législatifs [mais] un clivage s'est créé entre Bucarest, avec sa pléthore de séminaires consacrés aux droits de l'homme, à la démocratie, aux chances égales pour les femmes, et le reste du pays (...). Nous parlons souvent au nom des autres (...), nous lançons des stratégies (...), nous transmettons souvent des messages non intériorisés, nous nous servons de termes et de concepts que nous ne sentons pas nôtres ${ }^{63}$ ».

\footnotetext{
${ }^{61}$ Dans la même optique, mais sur un terrain bien différent, un travail fort stimulant s'intéresse aux pratiques philanthropiques d'associations internationales ciblant des femmes noires des couches populaires en Afrique du Sud, dans le cadre des réformes démocratiques des années 1990. L'analyse historique des pratiques et des représentations met en lumière la reproduction de rapports sociaux de type colonial, perpétués sous couvert d' « aide au développement » et de soutien à l'« émancipation des femmes » (D. Mindry, 2001).

${ }^{62}$ L. Grünberg, 1998, p. 19.

${ }^{63}$ Idem, 1999, p. 28. Idem, 2008 pour de plus amples développements au même sujet. Pour des propos similaires, on peut se rapporter aux témoignages portant sur le terrain ex-Yougoslave dans M. Walsh, 1998 et A. Bagic, 2006, qui montrent que, en rupture avec le contexte social et culturel local, la rhétorique centrée sur la figure de la femme vulnérable, victime de la guerre fut pendant longtemps l'unique registre permettant de trouver une longueur d'onde commune avec les agences d'aide internationale.
} 
Etudier les mécanismes et les relais à travers lesquels sont définies et diffusées les thématiques jugées prioritaires pour la recherche sur le genre en Europe centrale et orientale suppose de restituer les processus sociaux et les enjeux politiques liés à la sélection des problématiques et des grilles théoriques mobilisées pour interpréter les expériences nationales et les réalités empiriques spécifiques. Le sens de la réflexion que nous proposons consiste donc à aborder les conditions sociales ayant conduit aux choix des sujets de recherche apparus comme possibles dans le contexte des pays postcommunistes, en sachant que, à côté de thèmes comme les minorités, l'ethnicité, le nationalisme, le genre fait partie des nouveaux domaines investis en priorité. Certains travaux portant sur les «democratization studies » montrent d'ailleurs que les sciences sociales peuvent fonctionner comme outils de domination et véhicules d'imposition de normes globales ${ }^{64}$. Seule une démarche au plus près du terrain, centrée sur l'analyse de quelques cas concrets, peut élucider le fonctionnement pratique des rapports noués dans le cadre de l'implantation des études féministes au sein des PECO.

\section{L’irrépressible banalisation du genre}

La sociologie de l'émergence des préoccupations académiques centrées sur le genre en Europe de l'est implique aussi une réflexion théorique et épistémologique sur les usages de la notion. Il s'agit d'abord de comprendre et de mettre en lumière ses avatars au moment de sa conversion en agenda politique global, à savoir lors de son inscription sur les programmes de développement ${ }^{65}$. La question fondamentale qui se pose est de savoir en quelle mesure un

\footnotetext{
${ }^{64} \mathrm{~N}$. Guilhot, cit.

${ }^{65}$ S. Roth, 2004, pp. 20-21, pour l'expression «gender as political agenda ». Voir aussi M. Snyder, 2006 qui critique l'utilisation de cette notion par les organismes supranationaux, au lieu de «femmes » et «féminisme ».
} 
concept originairement critique ${ }^{66}$ peut garder ses vertus heuristiques et échapper à la normalisation lorsqu'il devient l'objet d'usages bureaucratiques, approprié par des instances extérieures à l'espace scientifique.

Le paradigme du genre semble être importé en Europe centrale et orientale à la fois par les voies propres à la communication scientifique et par des relais bureaucratiques. Pour cette raison, il est légitime de se demander s'il garde ou non le sens réflexif qui lui était propre au moment de son entrée dans les sciences sociales ${ }^{67}$. L'appel à «prendre en compte le genre », à prétention universelle et englobante, ne conduit-il pas à masquer les spécificités historiques et géographiques des revendications et des luttes des femmes et/ou féministes ? Comment une grille unificatrice pourrait-elle arriver à restituer de façon nuancée les particularités des cas nationaux et la diversité des mobilisations ? Plus encore, il faut s'interroger sur la pertinence de certaines analyses entreprises à titre d'expertise sur le genre, qui n'utilisent le travail de terrain que pour y chercher les arguments servant à confirmer les idées établies d'avance - sur le statut de victimes des femmes, par exemple - et finissent par occulter tout phénomène n'entrant pas dans cette perspective.

Certaines spécialistes ont signalé les détournements, voire les pièges de l'inscription du genre sur les agendas bureaucratiques ${ }^{68}$. Au-delà de la perte du sens critique du concept, voire de sa «lobotomisation» et de sa transformation en «technologie de recherche de fonds », elles ont mis en évidence des effets pervers tels que le bureaucratisme, l'affaiblissement et la dépolitisation des revendications militantes féminines, l'uniformisation des problématiques, l'effacement ou la non prise en compte des manifestations spontanées et

\footnotetext{
${ }^{66}$ Dans son sens critique, le concept vise à décrire les rapports de pouvoir inscrits dans les rapports sociaux de sexes et à intégrer cette dimension dans la production de connaissances sur le monde social, cf. J. W. Scott, 1988, N.-C. Mathieu, 1991.

${ }^{67}$ Nous avons souligné ailleurs le processus de dépolitisation de la notion de «genre » dans les contextes postcommunistes, où elle est systématiquement utilisée pour camoufler et rendre recevable une revendication féministe qui ne peut pas dire pas son nom (I. Cîrstocea, 2009, à paraître). Voir aussi J. Butler, E. Fassin, J. Scott, 2007, pour des réflexions sur la «Macdonaldisation du genre » et les effets de mode qui entourent la notion, exportée rapidement et perdant son épaisseur théorique.

${ }_{68}$ J. W. Scott, 2001; 2004; J. Bisilliat (éd.), 2003; H. Hirata et alii, 2000 (notamment les chapitres « Mouvements féministes », « Mondialisation », « Développement »); A. Spehar, 2004 ; Kasic, 2004a ; 2004b.
} 
des mobilisations qui ne rentrent pas dans les catégories de lecture du monde social inscrites sur les programmes de gestion politique.

Certaines études quelque peu décalées par rapport au mainstream des recherches sur les PECO mettent en lumière des formes d'expression et d'action qui battent en brèche le consensus quant à l' « inertie » des femmes est-européennes. En Russie, par exemple, on a pu repérer des associations féminines constituées à travers une expérience sociale immédiatement partagée et des problèmes pratiques communs : il s'agit de groupes de mères de soldats ou d'enfants handicapés. D'après les critères communs, ils ne correspondent pas à la catégorie des mobilisations féministes mais ils fonctionnent toutefois sur la base d'une solidarité et d'une identité féminines assumées en tant que telles, mettent en avant les rôles maternels - donc des représentations traditionnelles de la division sociale des sexes - et adoptent une rhétorique de l'antipolitique qui ne retire rien à leur assertivité en tant qu'acteurs politiques $^{69}$. Prenant l'exemple des Mères de Prague, une association tchèque similaire, une sociologue praguoise très présente dans les échanges Est-Ouest au sujet du féminisme, exdissidente anticommuniste et co-fondatrice au début des années 1990 d'un centre d'études de genre, considère que les femmes de son pays préfèrent « résoudre des problèmes concrets » et non pas reprendre et diffuser des engagements féministes théoriques d'inspiration occidentale $^{70}$. Enfin, au sujet des thèmes sociaux qui ont suscité des mobilisations féminines, on peut évoquer une pétition contre un projet de loir sur l'augmentation de l'âge à la retraite en Hongrie ${ }^{71}$.

Sur la base de dispositifs d'observation comprenant des terrains contrastés, d'autres travaux montrent l'existence de formes d'action et d'expression (infra)politique des femmes à travers des canaux d'expression originaux. En République tchèque, par exemple, un espace de contestation de la division traditionnelle des sexes semble voir été ouvert par des médias de

\footnotetext{
${ }^{69}$ A. White 2000, 218 ; R. Kay, 2000 ; A. Hrycak, 2002.

${ }^{70}$ J. Siklova, 1997, 78.

${ }^{71}$ K. Fabian, 2006.
} 
consommation telles que les publications Harlequin et la revue Cosmopolitan, importées et adaptées au marché national dans le courant de la décennie 1990. Défiant l'image classique de cette presse, dénoncée depuis des lustres comme un site de reproduction des rapports traditionnels des sexes et d'une conception dominée du féminin ${ }^{72}$, elles constitueraient un espace d'expression pour un «féminisme de facto», apparemment bien plus efficace que celui promu par les institutions bureaucratiques, les ONG et les publications universitaires ${ }^{73}$.

Enfin, nous pouvons nous référer à notre propre étude sur un mouvement infrapolitique constitué autour du courrier des lectrices d'un magazine populaire roumain, Formula As, fondé par une femme écrivain dans les années 1990 : bien que difficilement audibles dans un espace public monopolisé par les discours abstraits de la « démocratisation » et de l' «égalité des chances », les échanges autour de cette publication cristallisent, toujours au nom des rôles «traditionnels » féminins (care givers, responsables de la survie biologique des familles, etc.), d'abord une solidarité effective aidant à dépasser les difficultés économiques immédiates des premières années postcommunistes, ensuite une ample protestation pour défendre un site écologique menacé, qui canalise en même temps une revendication identitaire nationale mise à mal par la globalisation économique ${ }^{74}$.

Si fragmentaires qu'ils soient, ces exemples montrent bien que, malgré l'opinion commune qui les voit comme inertes et inactives politiquement, les femmes est-européennes identifient leurs problèmes, ainsi que des terrains de mobilisation et d'expression. Dans un contexte caractérisé par des changements économiques difficiles à gérer au quotidien, elles arrivent rarement à « distiller » leurs revendications, à les traduire pour les faire entendre dans l'espace formalisé de la compétition politique et encore moins à les mouler dans des formes identifiables comme des engagements féministes, qui mettraient en avant et théoriseraient

\footnotetext{
72 A. M. Dardigna, 1978 ; M. Ferguson, 1983.

73 «...même si le féminisme est marginal dans la culture tchèque, il est plus que bien accueilli dans la version tchèque de Cosmopolitan » (J. True, 2003, 127-129, la traduction nous appartient).

${ }^{74}$ I. Cîrstocea, 2002a; 2006a; I. Cîrstocea, A. Heemeryck, 2005.
} 
éventuellement leur identité politique commune, voire les objectifs partagés de leurs combats sur le long terme. Le débat d'actualité des années 1990 étant notamment consacré à des thèmes comme la «démocratisation », l' «européanisation », la «lustration », «la corruption », etc., les sujets «mineurs » comme la gestion quotidienne des familles sont traités comme des faits divers tout au plus ${ }^{75}$ et c'est alors par l'invention de tribunes ad-hoc que le manque est partiellement comblé, comme le montrent les exemples que nous venons de citer.

Dès lors, en comparant, d'une part, les thématiques abordées par plusieurs centres de recherche de pays différents, et d'autre part les formes de mobilisation féminines spontanées qui ne se disent pas féministes et restent en dehors du mouvement des femmes officiellement reconnu comme tel, on peut envisager une étude des effets pervers de l'internationalisation du genre se manifestant à présent dans les PECO.

Ce n'est pas une conclusion à proprement parler qui va clore ce texte, mais une reprise synthétique des pistes repérées comme adéquates pour construire sociologiquement un objet d'étude centré sur l'émergence du nouveau domaine de connaissances intitulé «études sur le genre » ou «études féministes »dans les PECO. La sociologie critique du genre restant la toile de fond de cette démarche, on mettra en œuvre une approche pluridisciplinaire et on fera appel aux outils théoriques et méthodologiques de la sociologie des espaces scientifiques, de l'histoire sociale des sciences sociales, de l'histoire du féminisme et de la sociologie de la circulation internationale des idées. Soumis à l'épreuve d'une réflexion qui interrogera conjointement les legs historiques des régimes communistes et de l'émancipation socialiste des femmes, les logiques pratiques des transferts internationaux des acquis politiques et

\footnotetext{
${ }^{75}$ Cela va de pair avec l'ignorance des aspects sociaux des transformations par les travaux sur le postcommunisme, qui se concentrent sur les réformes politiques et économiques laissant de côté l'expérience des acteurs.
} 
scientifiques du féminisme, les facteurs rendant possible l'apparition et la consolidation de nouveaux domaines de production de savoir, le «féminisme académique » - dans les PECO » et ailleurs - perdra, certes, de l'air héroïque que lui attribuent ses coryphées. En revanche, on gagnera une vision moins «enchantée » du monde social - le cas échéant de l'espace des sciences sociales, ainsi que des mobilisations autour d'enjeux identitaires - et une nouvelle lumière sur le fonctionnement des champs de production intellectuelle dans une région du monde où les producteurs de savoirs semblent, aujourd'hui comme hier, avoir du mal à se détacher des « pouvoirs » en place.

\section{Références bibliographiques}

Muriel ANDRIOCCI et alii, 2003, «Premier recensement national des enseignements et des recherches sur le genre en France », Rapport final aux : Ministères des affaires sociales, du travail et de la solidarité, Ministère de la parité et de l'égalité professionnelle, Service des droits des femmes et de l'égalité, Ministère de l'éducation nationale, de l'enseignement supérieur et de la recherche, Ministère délégué de la recherche, ANEF (Association nationale des études féministes)

Peggy ANTROBUS, 2004, The Global Women's Movement. Origins, Issues and Strategies, Londres, New York, Zed Books

Aida BAGIC, 1997, "Bread and Roses », in Tanya RENNE (éd.), Ana's Land. Sisterhood in Eastern Europe, Boulder, Co. , Westview Press, p. 201 , 2006, «Women's Organizing in Post-Yougoslav Countries. Talking about 'Donors' », in Myra Marx FERREE, Aili Mari TRIPP (éds.), Global Feminism. Transnational Women's Activism, Organizing and Human Rights, New York, Londres, New York University Press, pp. 141-165

Mieke BAL, 2002, Travelling Concepts in the Humanities : a Rough Guide, Toronto, University of Toronto Press

Ann BELLOWS, 2001, "The Praxis of Food Work in Poland », in Marianne DEKOVEN (éd.), Feminist Locations, Global and Local, Theory and Practice, New Brunswick, New Jersey, Londres, Rutgers University Press, pp. 229-256

Jeanne BISILLIAT (éd.), 2003, Regards de femmes sur la globalisation. Approches critiques, Paris, Karthala

Luc BOLTANSKI, "L'espace positionnel. Multiplicité des positions institutionnelles et habitus de classe", in Revue française de sociologie, 14, 1, 1973, pp. 3-26

Chiara BONFIGLIOLI, 2008, Belgrade 1978. Remembering the conference 'Drugarica Zena. Zensko Pitanje - Novi Pristup?/Comrade Woman. The Women's Question : A New Approach?' 30 years after, mémoire de master (MA), Université d'Utrecht (Faculty of Arts, Women's Studies Research Master “Gender and Ethnicity”), 2007-2008 
Ioana BORZA et alii, 2006, Cartea neagra a egalitatii de sanse intre femei si barbati in Romania (Le livre noir de l'égalité des chances pour les femmes et les hommes en Roumanie), Bucarest, Societatea de analize feministe AnA

Pierre BOURDIEU, 1984, Homo academicus, Paris, Minuit , 1997, Méditations pascaliennes, Paris, Seuil , 2002a, Sciences de la science et réflexivité, Paris, Raisons d'agir 2002b, «Les conditions sociales de la circulation internationale des idées », in Actes de la recherche en sciences sociales, 145, pp. 3-8

Rosi BRAIDOTTI, 1997, «Uneasy Transitions », in Joan W. SCOTT et alii (dir.), Transitions, Environments, Translations. Feminism in International Politics, New York, Londres, Routledge, pp. 355-372 2002, «The Uses and Abuses of the Sex/Gender Distinction in European Feminist Practices », in Rosi BRAIDOTTI, Gabriele GRIFFIN (éds.), Thinking Differently. A Reader in European Women's Studies, Londres, Zed Books, pp. 285-307

, 2004, «Introduction: Gender Education for Active Citizenship and for the Pursuit of Excellence in Research », in Rosi BRAIDOTTI et alii (éds.), The Making of European Women's Studies, ATHENA, Université d'Utrecht, V, pp. 10-16

, Gabriele GRIFFIN (éds.), 2002, Thinking Differently. A Reader in European Women's Studies, Londres, Zed Books

Rosi BRAIDOTTI et alii, 1995, «Les études féministes en Europe », in ERASMUS évaluation de la coopération par domaines d'étude, section IX, Network of Interdisciplinary Women's Studies in Europe, Université d'Utrecht

, (éds.), 2000-, The Making of European Women's Studies. A work in progress report on curriculum development and related issues in gender education and research, ATHENA (Advanced Thematic Network in Activities in Women's Studies in Europe, Socrates Programme, European Commission), Université d'Utrecht

Laure BUSHEIKIN, 1997, «Is Sisterhood Really Global? Western Feminism in Eastern Europe », in Tanya RENNE (éd.), Ana's Land. Sisterhood in Eastern Europe, Boulder, Co., Westview Press, pp. 12-21

Judith BUTLER, Eric FASSIN, Joan W. SCOTT, 2007, «Pour ne pas en finir avec le genre » (entretien), in Sociétés \& Représentations, 24, pp. 285-306

Thomas CAROTHERS, 2004, Critical Mission. Essays on Democracy Promotion, Washington DC, Carnegie Endowment for International Peace

Allaine CERWONKA, 2008, « Traveling Feminist Thought : 'Difference' and Transculturation in Central and Eastern European Feminism », in Signs. Journal of Women in Culture and Society, 33, 4, pp. 809-832

Ioana CÎRSTOCEA, 2002a, «Formula As: la parole politique des sans voix », in Rose-Marie LAGRAVE et alii (éds.), Dissemblances. Jeux et enjeux du genre, Paris, L'Harmattan, pp. 225-238

2002b, «Inventer un acteur politique: le régime communiste roumain et la 'question femme' (1945-1965) », in Revue des études sud-est européennes, 40, 1-2, pp. 233256

,2003, «Le devoir féminin entre norme communiste et pratique quotidienne. Une étude de cas », in Alexandra IONESCU, Odette TOMESCU-HATTO (éds.), Politique et société dans la Roumanie contemporaine, Paris, L'Harmattan, pp. 25-50

, 2004a, «Contribution à une sociologie de la 'transition' roumaine à travers le prisme de la condition féminine et des représentations de la féminité », thèse de doctorat en sociologie, EHESS Paris 
,2004b «L'enjeu du genre sur le marché intellectuel roumain postcommuniste », in Transitions, XLIV, 1 («L'égalité des sexes en Europe centrale et orientale : entre espoir et déconvenues $\gg)$, pp. 165-177

2006a, Faire et vivre le postcommunisme. Les femmes roumaines face à la «transition », Bruxelles, Editions de l'université de Bruxelles

, 2006b, «Présences du passé dans les nouvelles mobilisations pour la cause des

femmes en Roumanie », in Sandrine KOTT, Martine MESPOULET, Antoine ROGER (éds.), Le postcommunisme dans l'histoire, Bruxelles, Editions de l'université de Bruxelles, pp. 151162

_. 2008, «'Between the Past and the West' : le dilemme du féminisme en Europe de l'est postcommuniste », in Sociétés contemporaines, 71, 3, pp. 7-27

, 2009, «Du 'genre' critique au 'genre' neutre : effets de circulation », in

Bérengère MARQUES-PERREIRA, Petra MEIER, David PATTERNOTTE (éds.), Actes du Groupe "Genre », IVe Congrès de l'Association belge de science politique (Louvain-laNeuve, avril 2008)» (à paraître)

, Antoine HEEMERYCK, 2005, «Occurrences paradoxales du politique en

Roumanie », in Journal des anthropologues, 102-103, pp. 371-409

Dominique COLAS (éd.), 2002, L'Europe postcommuniste, Paris, PUF

Anne-Marie DARDIGNA, 1978, Femmes-femmes sur papier glacé, Paris, Maspero

Manisha DESAI, 2005, «Le transnationalisme: nouveau visage de la politique féministe depuis Beijing », in Revue internationale des sciences sociales, 184, pp. 349-361

Otilia DRAGOMIR, Mihaela MIROIU (éds.), 2002, Lexicon feminist (Dictionnaire féministe), Iaşi, Polirom

Barbara EINHORN, 1993, Cinderella Goes to Market. Citizenship, Gender and Women's Movements in East Central Europe, Londres, New York, Verso 2002, «Focusing on Gender Equality Worldwide», in Eniko MAGYARIVINCZE (éd.), Talking Feminist Institutions. Interviews with Leading European Scholars, Cluj, Fondation Desire, pp. 77-94

European Network for Women's Studies (ENWS), 1993, Establishing Gender Studies in Central Eastern European Countries. Workshop, Wassenaar, 5-8 novembre 1992 (ronéo)

Katalin FABIAN, 2006, "Bridges across the Public-Private Divide. The WelfareRelated Activism of Hungarian Women after 1989 ", in Edith SAURER et alii (éds.), Women's Movements. Networks and Debates in Post-Communiste Countries in the 19th and 20th Centuries, Cologne, Weimar, Vienne, Bohlau Verlag, pp. 47-64

Marjorie FERGUSON, 1983, Forever Feminine. Women's Magazines and the Cult of Femininity, Londres, Heinemann

Myra Marx FERREE, 1995, «Patriarchies and Feminism: The Two Women's Movements of Post-Unification Germany», in Social Politics. International Studies in Gender, State and Society, 2, 1, pp. 10-24

, 2006, «Globalization and Feminism: Opportunities and Obstacles for Activism in the Global Arena », in Myra Marx FERREE, Aili Mari TRIPP (éds.), Global Feminism. Transnational Women's Activism, Organizing and Human Rights, New York, Londres, New York University Press, pp. 3-23

et alii, 2001, «Constructing Global Feminism: Transnational Advocacy Networks and Russian Women's Activism », in Signs. Journal of Women in Culture and Society, 26, 4, pp. $1155-1186$

, Aili Mari TRIPP (éds.), 2006, Global Feminism. Transnational Women's Activism, Organizing and Human Rights, New York, Londres, New York University Press

Maxime FOREST, Georges MINK (éds.), Postcommunisme: les sciences sociales à l'épreuve, Paris, L'Harmattan, 2004 
Mihaela FRUNZA, Teodora VACARESCU (éds.), 2004, Gender and the (Post) East/West Divide, Cluj-Napoca, Limes

Nanette FUNK, 1993, «Feminism East and West », in Nanette FUNK, Magda MUELLER (éds.), Gender Politics and Post-Communism. Reflections from Eastern Europe and the Former Soviet Union, New York, Londres, Routledge, pp. 331-337

, 2006, «Women's NGOS in Central and Eastern Europe and the Former Soviet Union: The Imperialist Criticism », in Jasmina LUKIC et alii (éds.), Women and Citizenship in Eastern Europe, Aldershot, Ashgate, pp. 265-287

, 2007, «Fifteen Years of the East-West Women's Dialogue », in Janet Elise JOHNSON, Jean C. ROBINSON (éds.), Living Gender after Communism, Bloomington, Indianapolis, Indiana University Press, pp. 203-226

Magda MUELLER (éds.), 1993, Gender Politics and Post-Communism. Reflections from Eastern Europe and the Former Soviet Union, New York, Londres, Routledge

Malgorzata FUSZARA, 2000, «Feminism, the New Millenium, and Ourselves: A Polish View », in Signs. Journal of Women in Culture and Society, 25, 4, 2000, pp. 10691075

Susan GAL, 2003, « Movements of Feminism. The Circulation of Discourses about Women », in Barbara HOBSON (éd.), Recognition Struggles and Social Movements. Contested Identities, Power and Agency, Cambridge, New York, Cambridge University Press, pp. 93-120

, Gail KLIGMAN (éds.), 2000a, Reproducing Gender. Politics, Publics and Everyday Life after the Socialism, Princeton, New Jersey, Princeton University Press

University Press 2000b, The Politics of Gender after Socialism, Princeton, New Jersey, Princeton

Gender Studies \&Women's Studies Directory. Resources in the Countries of Central and Eastern Europe, the former Soviet Union and Mongolia, Open Society Institute, Network Women's Program, Budapest, 1999

Gabriela GHEBREA et alii, 2005a, Politici de gen în România. Două studii de caz (Politiques du genre en Roumanie. Deux études de cas), Bucarest, Nemira ,2005b, Mapping Romanian Women's Campaign for Change, Bucarest, Nemira

Mihai Dinu GHEORGHIU, 2004, «Les centres européens d'excellence dans les sciences sociales et humaines ", in Catherine DURANDIN, Magda CARNECI (éds.), Perspectives roumaines. Du postcommunisme à l'intégration européenne, Paris, L'Harmattan, pp. 139-162

Kristen GHODSEE, K., 2004, «Feminism-by-Design: Emerging Capitalisms, Cultural Feminism, and Women's NGOs in Post-socialist Eastern Europe », Signs : Journal of Women in Culture an d Society, 29, 3, pp. 727-753

2006, « Nongovernmental Ogres? How Feminist NGOs Undermine Women in Post-socialist Eastern Europe », in The International Journal of Non-for-Profit Law, 8, 3, pp. 43-59

Gabrielle GRIFFIN, 2004, «Employment and Women's Studies : The impact of Women's Studies Training on Women's Employment in Europe », in Rosi BRAIDOTTI et alii (éds.), The Making of European Women's Studies, V, pp. 121-140

(éd.), 2005, Doing Women's Studies: Employment Opportunities, Personal Impacts and Social Consequences, Londres, New York, Zed Books

Laura GRÜNBERG, 1998, « Tablou cu femei lucrând la tranziţie » (Tableau : femmes oeuvrant à la transition), in AnAlize, revistă de studii feministe, 1-2, pp. 16-25

1999, «De la Bucureşti la Buteni sau lungul drum al politicilor sociale către femei » (De Buteni à Bucarest, le long chemin des politiques sociales vers les femmes), in 
Liliana POPESCU (éd.), Gen şi politică. Femeile din România în viaţa publică (Genre et politique. Les femmes roumaines dans la vie publique), Bucarest, Société d'analyses féministes AnA, PNUD, pp. 27-41

,2000a, «Women's NGOs in Romania », in Susan GAL, Gail KLIGMAN (éds.), Reproducing Gender. Politics, Publics and Everyday Life after the Socialism, Princeton, New Jersey, Princeton University Press, pp. 307-336

2000b, «ONGizarea feminismului în România. Eşecul unui succes » (L'ONGisation du féminisme en Roumanie. L'échec d'un succès), AnAlize, revistă de studii feministe, 7, p. 14-19

Eastern Europe, Bucarest, UNESCO-CEPES

2008, biONGrafie. AnA - istoria traita a unui ONG de femei (biONGraphie. AnA

- histoire vécue d'une ONG de femmes), Iasi, Polirom, 2008

Eliane GUBIN et alii, 2004, Le siècle des féminismes, Paris, Editions de l'Atelier/Editions Ouvrières

Nicolas GUILHOT, 2001, «Les professionnels de la démocratie. Logiques militantes et logiques savantes dans le nouvel internationalisme américain », in Actes de la recherche en sciences sociales, 139 , pp. 53-66

2004, «Une vocation philanthropique. George Soros, les sciences sociales et la régulation du marché mondial », in Actes de la recherche en sciences sociales, 151-152, pp. $37-48$

2005, The Democracy Makers: Human Rights and International Order, New York, Columbia University Press

2007, «Reforming the World: George Soros, Global Capitalism and the Philanthropic Management of the Social Sciences », in Critical Sociology, 33, pp. 447-477

Chris. M. HANN (éd.), 2002, Post-socialism. Ideals, Ideologies and Practices in Eurasia, Londres, New York, Routledge

Johan HEILBRON, Rémi LENOIR, Gisèle SAPIRO (éds.), 2004, Pour une histoire des sciences sociales. Hommage à Pierre Bourdieu, Paris, Fayard

Johan HEILBRON, Nicolas GUILHOT, Laurent JEANPIERRE, 2009, «Vers une histoire transnationale des sciences sociales », in Sociétés contemporaines, 1, 73, pp. 121-145

Helena HIRATA et alii, 2000, Dictionnaire critique du féminisme, Paris, PUF (nouvelle édition 2004)

Bernard HOURS, Monique SELIM, 2003, Solidarités et compétences. Idéologies et pratiques, Paris, L'Harmattan

Alexandra HRYCAK, «From Mothers' Rights to Equal Rights. Post-Soviet Grassroots Women's Associations », in Nancy A. NAPLES, Manisha DESAI, Women's Activism and Globalization. Linking Local Struggles and Transnational Politics, Londres, New York, Routledge, 2002, pp. 64-82

http://www.zenskestudie.edu.yu (consulté le 28.12.2004)

Sophie JACQUOT, 2006, L'action publique communautaire et ses instruments. Les politiques d'égalité entre les femmes et les hommes à l'épreuve du gender mainstreaming, thèse de doctorat, IEP Paris

Journal des anthropologues («Parcours de l'ethnologie dans le monde postsoviétique »), 87, 2001

Journal des anthropologues («Les ONG : médiations politiques et globalisation»), 94-95, 2003

Biljana KASIC, 2004a, «Women's Studies in Croatia : Between Feminist Sensibility and Critical Responsibility », in Rosi BRAIDOTTI et alii (éds.), The Making of European Women's Studies, ATHENA, V, pp. 30-40 
, 2004b, «Féminisme-s 'Est-Ouest' dans une perspective postcoloniale », in Nouvelles Questions Féministes («Postcommunisme : genre et Etats en transition »), 23, 2, pp. 78-86

Rebecca KAY, 2000, "'It's about Helping Women to Believe in Themselves': Grassroots Women's Organizations in Contemporary Russian Society », in Anna BULL et alii (éds.), Feminisms and Women's Movements in Contemporary Europe, Londres, Macmillan Press LTD, Houndmills, Basingstoke, Hampshire, New York, St. Martin's Press, LLC, pp. 228-243

Sandrine KOTT et alii (éds.), 2006, Le postcommunisme dans l'histoire, Bruxelles, Editions de l'Université de Bruxelles

Rose-Marie LAGRAVE, 1998, Voyage aux pays d'une utopie déchue : plaidoyer pour l'Europe centrale, Paris, PUF

Sabine LANG, 1997, «The NGOization of Feminism. Institutionalization and Institution Building within the German Women's Movements », in Joan W. SCOTT et alii (éds.), Transitions, Environments, Translations. Feminism in International Politics, New York, Londres, Routledge, pp. 101-120

Vera LITRICHIN, Lepa MLADENOVIC, 1997, «Belgrade Feminists : Separation, Guilt and Identity Crisis », in Tanya RENNE (éd.), Ana's Land. Sisterhood in Eastern Europe, Boulder, Co., Westview Press pp. 176-185

Zsofia LORAND, 2007, Feminism as Counterdiscourse in Yugoslavia in Two Different Contexts, Budapest, Central European University (mémoire de master, History Department)

Jasmina LUKIC et alii (éds.), 2006, Women and Citizenship in Eastern Europe, Aldershot, Ashgate

Nina LYKKE, 2004, «Women's Gender/Feminist Studies - a Post-disciplinary Discipline », in Rosi BRAIDOTTI et alii (éds.), The Making of European Women's Studies, , ATHENA, V, pp. 91-101

Eniko MAGYARI-VINCZE (éd.), 2002, Talking Feminist Institutions. Interviews with Leading European Scholars, Cluj, Fondation Desire

Nicole-Claude MATHIEU, 1991, L'anatomie politique. Catégorisations et idéologies du sexe, Paris, Côté-femmes éditions

Deborah MINDRY, 2001, «Nongovernmental Organizations, 'Grassroots' and the Politics of Virtue », in Signs. Journal of Women in Culture and Society, 26, 4, pp. 1187-1211

Michael MINTROM, Jacqui TRUE, 2001, «Transnational Networks and Policy Diffusion: The Case of Gender Mainstreaming », International Studies Quarterly, 45, 1, pp. 27-57

Mariola MISIOROWSKA, 2004, «Le mouvement des femmes en Pologne postcommuniste et les acteurs internationaux », Recherches féministes, 17, 2, pp. 43-84

Francine MUEL-DREYFUS, 1996, Vichy et l'éternel féminin. Contribution à une sociologie politique de l'ordre des corps, Paris, Seuil

Ralitsa MUHARSKA, 2005, "Silences and Parodies in the East-West Dialogue », L'Homme. Z.F.G., 16, 1, pp. 36-47

Nancy A. NAPLES, Manisha DESAI, 2002, Women's Activism and Globalization. Linking Local Struggles and Transnational Politics, Londres, New York, Routledge

Nouvelles Questions Féministes («Postcommunisme : genre et Etats en transition »), 23, 2, 2004

Laurie OCCHIPINTI, 1996, « Two Steps Back? : Anti-Feminism in Eastern Europe », in Anthropology Today, 12, 6, pp. 13-18

Andrea PETO, 2001, "Conservative and Extreme Right-Wing Women in Contemporary Hungary. An Ideology in Transition », in Durda KNEZEVIC et alii, Women 
and Politics : Women and History, History without Women, Zagreb, Zenska Infoteka, pp. 265276

Andrea PETO (éd.), 2006, Teaching Gender Studies in Hungary, Budapest, Ministère de la Jeunesse, de la famille, des affaires sociales et de l'égalité des chances

Gautier PIROTTE, 2003, Une société civile postrévolutionnaire. Etude du nouveau secteur ONG en Roumanie : le cas de Iasi, Bruxelles, Académia Bruylant

Politique européenne ( « Genre et action publique en Europe »), 20, 2006

Anastasia POSADSKAYA-VANDERBECK, 1997, «On the Treshold of the Classroom. Dilemmas for Post-Soviet Russian Feminism », in Joan W. SCOTT et alii (éds.), Transitions, Environments, Translations. Feminism in International Politics, New York, Londres, Routledge, pp. 373-381

Nadège RAGARU, 2000-2001, «Les avatars de l'aide internationale dans les Balkans », in Revue internationale et stratégique, 40, pp. 49-66

Tanya RENNE (éd.), 1997, Ana's Land. Sisterhood in Eastern Europe, Boulder, Co., Westview Press

Revue d'histoire des sciences humaines («Quelle sociologie derrière le 'rideau de fer'? 1950-1989»), 14, 2007

Revue française de science politique («Les transitions démocratiques. Regards sur l'état de la 'transitologie' »), 50, 4-5, 2000

Silke ROTH, 2004, «One Step Forwards, One Step Back, One Step Forwards. The Impact of EU Policy on Gender Relations in Central and Eastern Europe », in Transitions, XLIV, 1, pp. 15-27

, 2007, «Sisterhood and Solidarity? Women's Organizations in the Expanded European Union », in Social Politics : International Studies in Gender, State and Society (« Gender, Civil Society, and Participation »), 14, 4, pp. 460-487

Edith SAURER et alii (dir.), 2006, Women's Movements. Networks and Debates in Post-Communiste Countries in the 19th and 20th Centuries, Cologne, Weimar, Vienne, Bohlau Verlag

Joan W. SCOTT, 1988, Gender and the Politics of History, New York, Columbia University Press 1999, «Some Reflections on Gender and Politics », in Myra Marx FERREE et alii (éds.), Revisioning Gender, Londres, New Delhi, Sage Publications, pp. 70-96 2001, «Millenial Fantasies. The Future of 'Gender' in the $21^{\text {st }}$ Century », in Caroline ARNI, Claudia HONEGGER (éds.), Gender - die Tucken einer Kategorie. Joan W. Scott, Geschichte und Politik, Zurich, Chronos Verlag, pp. 19-37 2004, «Feminist Reverberations », in Durda KNEZEVIC et alii, Women and Politics : Contemporary Women's/Feminist Movements in Post-Communist Countries - 10 Years After, Zagreb, Zenska Infoteka, pp. 179-197 et alii (éds.), 1997, Transitions, Environments, Translations. Feminism in International Politics, New York, Londres, Routledge

Signs. Journal of Women in Culture and Society (« Eastern Europe »), 29, 3, 2004

Kornelia SLAVOVA, 2006, « Looking at Western Feminisms through the Double Lens of Eastern Europe and the Third World », in Jasmina LUKIC et alii (éds.), Women and Citizenship in Central and Eastern Europe, Aldershot, Ashgate, pp. 245-263

Amanda SLOAT, 2005, «The Rebirth of Civil Society. The Growth of Women's NGOs in Central and Eastern Europe », in The European Journal of Women's Studies, 12, 4, pp. 437-452

Ann SNITOW, 1995, "Feminist Future in the Former East Bloc », in Marina BLAGOJEVIC et alii (éds.), What Can We Do For Ourselves? East-European Feminist 
Conference, Belgarde, Center for Women's Studies, Research and Communication, pp.141155 , 2006, "Cautionary Tales », in Jasmina LUKIC et alii (éds.), Women and Citizenship in Central and Eastern Europe, Aldershot, Ashgate, pp. 287-297

Margaret SNYDER, «Unlikely Grandmother: The UN and the Global Women's Movement ", in Myra Marx FERREE, Aili Mari TRIPP (éds.), Global Feminism. Transnational Women's Activism, Organizing and Human Rights, New York, Londres, New York University Press, pp. 24-50

Social Politics. International Studies in Gender, State, and Society (Special Issue on Gender Mainstreaming), 12, 3, 2005

Andrea SPEHAR, 2004, «Research on Contemporary Women's Movements and Gender Policy in Post-Communist Countries - Shortcommings and Opportunities », in Durda KNEZEVIC et alii, Women and Politics : Contemporary Women's/Feminist Movements in Post-Communist Countries - 10 Years After, Zagreb, Zenska Infoteka, pp. 145-154 2005, «Eastern European Women, Winners or Losers in Post-Communist Transitions ? », http://hdl.handle.net/2077/517 (consulté le 20.02.2007)

Virgil STOICA, 2003, «Public Policies Concerning the Equality of Opportunities between Women and Men, 1990-2000 », in Romanian Journal of Society and Politics, 3, 1, pp. 192-232

Julia SZALAI, 1991, «Some Aspects of Changing Situation of Women in Hungary », in Signs. Journal of Women in Culture and Society, 16, 1, pp. 152- 170 1996, Two Studies on Changing Gender Relations in Post-1989 Hungary, Collegium Budapest, Discussion Paper no. 30, July

Transitions («L'égalité des chances en Europe centrale et orientale : entre espoir et déconvenues »), XLIV, 1, 2004

Jacqui TRUE, 2003, Gender, Globalization, and Post-socialism : the Czech Republic after Communism, New York, Chichester, West Sussex, Columbia University Press

Mieke VERLOO, 2002, "On the Bridge Between Science and Policy Making », in Eniko MAGYARI-VINCZE (éd.), Talking Feminist Institutions. Interviews with Leading European Scholars, Cluj, Fondation Desire, pp. 95-116

Martha WALSH, 1998, "Mind the Gap. Feminist Theory Failed to Meet Development Practice. A Missed Opportunity in Bosnia and Herzegovina », in The European Journal of Women's Studies, 5, 3-4, pp. 329-343

Georgina WAYLEN, 1994, «Women and Democratization : Conceptualizing Gender Relations in Transition Politics », in World Politics, 46, 3, pp. 327-354

Janine R. WEDEL, Collision and Collusion. The Strange Case of Western Aid to Eastern Europe 1989-1998, New York, St. Martin's Press, 1998

Elaine Susan WEINER, 2004, «Imperfect Vision : Failing to See the 'Difference' of Central and East European Women », in Mihaela FRUNZA, Teodora Eliza VACARESCU (éds.), Gender and the (Post) East/West Divide, Cluj-Napoca, Limes, pp. 27-50

Michael WERNER, Bénédicte ZIMMERMANN, 2004, «Penser l'histoire croisée : entre empirie et réflexivité », in Idem (éds.), De la comparaison à l'histoire croisée, Paris, Seuil, pp. 15-50

Anna WHITE, 2000, "New Mothers' Campaigning Organizations in Russia », in Anna BULL et alii (éds.), Feminisms and Women's Movements in Contemporary Europe, Londres, Houndmills, Basingstoke, Hampshire, Macmillan Press LTD, New York, St. Martin's press, LLC, pp. 211-227

Veronika WOHRER, 2004, « Border Crossers. Gender Discourses between 'East' and

'West' », in Mihaela FRUNZA, Teodora Eliza VACARESCU (éds.), Gender and the (Post) East/West Divide, Cluj-Napoca, Limes, pp. 51-60 
Elena ZAMFIR, 1999, «Problematica femeii pe agenda politică a tranziţiei » (La problématique de la femme sur l'agenda politique de la transition), in Raportul naţional al dezvoltării umane (Rapport national du développement humain), Bucarest, PNUD, pp. 70-80

Susan ZIMMERMANN, 2007, The Institutionalization of Women and Gender Studies in Higher Education in Central and Eastern Europe and the Former Soviet Union : Assymetric Politics and the Regional-Transnational Configuration, http://www.duke.edu/womstud/Budapest.html (consulté le 5.07.2007) 


\section{Résumé}

Le domaine de recherche et d'enseignement des women's/gender studies s'est institutionnalisé pendant la décennie 1990 dans les pays de l'Europe centrale et orientale (PECO). Les travaux produits dans ce cadre fonctionnent souvent à des fins d'expertise, se donnent pour but de contribuer à améliorer la condition des femmes et se situent au cœur des programmes de recomposition sociale et politique à la sortie du communisme. Les agents qui en sont porteurs investissent simultanément des espaces sociaux contrastés (scientifique, associatif et politique) et sont des figures exemplaires du féminisme multipositionnel de la mondialisation. Se trouvant au croisement de logiques savantes et militantes, nationales et internationales, ces études sont un révélateur de la refonte des sciences sociales de l'émergence de nouveaux champs d'interrogations, de la circulation internationale et de l'importation de problématiques et préoccupations scientifiques, enfin, des recompositions des élites savantes dans les PECO. L'article s'ouvre par une reconstitution du contexte d'émergence des recherches sur le genre en Europe de l'est dans les années 1990, caractérisé à la fois par la dégradation de la condition féminine et le rejet du féminisme en réaction au surinvestissement des régimes socialistes sur les femmes. Un plan rapproché sur le cas de la Roumanie soutient une réflexion sur les enjeux de la «question femme » dans le cadre de la «transition », lorsqu'elle est investie par une série d'agents engagés dans les réformes pour la démocratisation qui s'approprient la préoccupation politique transnationale pour les droits des femmes. Enfin, sur la base de ces préliminaires, quelques directions de recherche et hypothèses provisoires sont esquissées : sociologie des gender studies en tant que nouvelle discipline académique, dans une perspective inspirée de l'histoire sociale des sciences sociales; sociologie de la circulation internationale des idées féministes et des logiques pratiques à l'œuvre dans les échanges intellectuels Est-Ouest; réflexion sur les effets de la prolifération des usages de la notion de «genre » en dehors des milieux académiques et son appropriation par des instances bureaucratiques.

\section{Mots-clés}

Europe de l'est ; postcommunisme ; féminisme ; genre ; espace académique

\section{Notice bio-bibliographique}

Ioana CIRSTOCEA est chargée de recherche au CNRS (PRISME-GSPE, Strasbourg) depuis 2006. Ancienne élève de l'Ecole doctorale en sciences sociales (Bucarest) et de l'Ecole des Hautes Etudes en Sciences Sociales (Paris) où elle a obtenu son doctorat en sociologie en 2004, elle est notamment l'auteure de Faire et vivre le postcommunisme. Les femmes roumaines face à la 'transition', Bruxelles, Editions de l'université de Bruxelles, 2006. Elle a aussi publié des articles dans Revue des études sud-est européennes (2002, 2005), Transitions (2002, 2004), Journal des anthropologues (2005), Revue d'études comparatives Est-Ouest (2006), Revue d'histoire des sciences sociales (2007), Sociétés contemporaines (2008) et de contributions à plusieurs volumes collectifs.

Adresse postale : 17, rue de Cadix, 75015 Paris, France

Contact : Ioana.Cîrstocea@misha.fr; ioana_cirstocea@hotmail.com; tél. : 0033617142412 